\title{
Propiedades mécanicas y tribólogicas de recubrimientos alumina/ titania proyectados por oxifuel (spray llama)
}

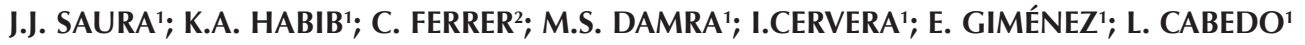 \\ 1 Departamento de Ingeniería de Sistemas Industriales y Diseño, Área de Ciencia de los Materiales e Ingeniería Metalúrgica. Universidad \\ Jaume I: 12071 Castellón. \\ 2 Departamento de Ingeniería Mecánica y de Materiales, UPV- Valencia
}

\begin{abstract}
En este trabajo se ha estudiado, las fases, las propiedades mecánicas y la resistencia al desgaste abrasivo de recubrimientos cerámicos alumina/titania proyectados por el proceso de oxifuel (spray llama). La proporción de titania tiene una fuerte influencia sobre la porosidad de los recubrimientos, habiéndose observado una disminución casi-lineal de la porosidad con el incremento de titania. Las fases cristalinas que resultan después de la proyección han variado según la naturaleza del polvo y el proceso térmico experimentado. Mientras la dureza obtenida depende sólo del porcentaje en peso de titania, la tenacidad es una función inversa de la dureza, y, la resistencia al desgaste por abrasión es una función creciente con la dureza de los recubrimientos.
\end{abstract}

Palabras clave: Alúmina-titania; recubrimientos cerámicos. spray llama, propiedades mecánicas, desgaste abrasivo.

Mechanical and tribological properties of alumina/titania coatings deposited by Ooxifuel.

In this paper the porosity, phases, mechanical properties and abrasive wear resistance of ceramic layers of $\mathrm{Al}_{2} \mathrm{O}_{3} / \mathrm{TiO}_{2}$ deposited using the flame spray process are evaluated.

The proportion of titania has a strong influence on the porosity of the coating, having observed an approximately linear plot decrease with the increasing of titania's content.

Crystalline phases of the deposited layers changed according to the characteristics of the powder and the thermal process employed. It has been observed that hardness depends only on the titania percentage, also toughness decreases with coatings hardness but resistance to abrasive wear traces an increasing lineal plot with this property.

Keywords: Alumina-titania; ceramic coating, flame spray, mechanical properties, abrasive wear.

\section{INTRODUCCIÓN}

Los recubrimientos alumina/titania son excelentes candidatos para aplicaciones contra el desgaste y la abrasión, así mismo tienen excelente resistencia a corrosión tanto galvánica como a altas temperaturas. Tales recubrimientos encuentran aplicaciones en manguitos protectores de ejes, fundas de termopares, aislantes eléctricos, ejes de bombas, etc, y allí donde haya que combinar alta resistencia al desgaste, bajo coeficiente de rozamiento y elevadas temperaturas de servicio. Usualmente estos recubrimientos son aplicados mediante procesos de plasma spray (PS) que consideran necesario fundir la partículas de polvo cerámico, o mediante técnicas de oxifuel de alta velocidad (HVOF) aunque en menor proporción (1).

La técnica de proyección por oxifuel, flame spray (OF), tiene desventajas respecto a las PS o HVOF en cuanto que las estructuras obtenidas son de mayor tamaño de grano, así como las grietas y poros; pero tiene la ventaja de ser una técnica mas económica, menos dificultosa en su manejo y más adaptable a los procesos de fabricación de reducido numero de unidades o de recuperación de piezas. La técnica de oxyfuel moderna permite alcanzar velocidades de partículas proyectadas hasta unos $300 \mathrm{~m} / \mathrm{s}$ y temperaturas alrededor de $3000^{\circ} \mathrm{C}(2-3)$.

En este trabajo se han investigado tres recubrimientos con diferentes proporciones de alúmina/titania usando la técnica de $\mathrm{OF}$ en condiciones optimizadas de trabajo. Se ha determinado la dureza, la tenacidad por indentación, y la resistencia a la abrasión frente a CSi. La resistencia a la abrasión se ha medido experimentalmente y se ha relacionado con la dureza.

El material base elegido ha sido acero inoxidable austenítico AISI 304, que reúne buena resistencia a oxidación a altas temperaturas y resistencia mecánica.

Se han utilizado chapas de $2 \mathrm{~mm}$ de espesor y varillas de $8 \mathrm{~mm}$ de diámetro; en ambos casos el material se ha suministrado en estado de temple austenítico (hipertemple). 
TABLA I. CONDICIONES DE PROYECCION.

\begin{tabular}{|c|c|c|c|c|c|c|c|c|c|c|c|}
\hline Muestra & $\begin{array}{c}\text { Velocidad } \\
\text { de la pistola } \\
(\mathrm{mm} / \mathrm{seg})\end{array}$ & Aleación & $\begin{array}{l}\mathrm{N}^{\circ} \text { de pasadas de } \\
\text { precalentamiento }\end{array}$ & $\begin{array}{l}\mathrm{N}^{\mathrm{o}} \text { de pasadas } \\
\text { de proyección }\end{array}$ & $\begin{array}{c}\text { Caudal } \\
\text { (posición) } \\
\left(^{*}\right)\end{array}$ & $\begin{array}{l}\text { Distancia de } \\
\text { proyección } \\
(\mathrm{mm})\end{array}$ & $\begin{array}{l}\text { Presión de } \\
\text { acetileno } \\
\text { (bar) }\end{array}$ & $\begin{array}{l}\text { Prersión } \\
\text { de } \\
\text { oxigeno } \\
\text { (bar) }\end{array}$ & $\begin{array}{c}\text { Presión } \\
\text { del aire } \\
\text { (bar) }\end{array}$ & $\begin{array}{c}\text { Tipo de } \\
\text { llama } \\
\left({ }^{* *}\right)\end{array}$ & $\begin{array}{c}\text { Espesor } \\
(\mu \mathrm{m})\end{array}$ \\
\hline A & 70 & Rototec 51000 & 1 & 1 & 2 & 150 & 0.7 & 3 & 1.5 & Neutra & 195 \\
\hline B & 70 & Rototec 51000 & 1 & 1 & 2 & 150 & 0.7 & 3 & 1.5 & Neutra & 160 \\
\hline $\mathrm{C}$ & 70 & Rototec 51000 & 1 & 1 & 2 & 150 & 0.7 & 3 & 1.5 & Neutra & 175 \\
\hline$\overline{\mathrm{A}}$ & 70 & 28020 & 0 & 5 & 1 & 85 & 0.8 & 4 & 3 & Neutra & 570 \\
\hline $\mathrm{B}$ & 70 & 28030 & 0 & 5 & 2 & 100 & 0.8 & 4 & 3 & Neutra & 575 \\
\hline $\mathrm{C}$ & 70 & 28060 & 0 & 5 & 2 & 100 & 0.8 & 4 & 3 & Neutra & 405 \\
\hline
\end{tabular}

$\left(^{*}\right)$ La pistola dispone de seis posiciones con caudales de polvos crecientes entre 1-6.

$\left.{ }^{(* *}\right)$ La llama neutra es aquella que no tiene exceso ni defecto de oxigeno. Su color es azul.

\section{RECUBRIMIENTOS}

El acero inoxidable austenítico de base ha sido preparado superficialmente por chorreado con abrasivo de corindón angular $(500 / 700 \mu \mathrm{m})$ a una presión de aire comprimido de 6 $\mathrm{Kg} / \mathrm{cm}^{2}$ durante 1 minuto.

Los recubrimientos constan de una primera capa de enganche y una segunda capa cerámica de acabado; ambos depósitos se han aplicado con un soplete spray llama CDS8000 de Castolin Eutectic (Suiza). La capa de enganche, Rototec 51000, también de Castolin, en un polvo comercial de tamaño de partículas entre $40 / 100 \mu \mathrm{m}$ y composición 95\% Ni; $5 \% \mathrm{Al}$ en peso, y es utilizado como capa intermedia entre sustratos de base férrea, base cobre y base aluminio, y aleaciones de acabado base hierro, base cobre, base níquel y aleaciones cerámicas.

Sobre la capa de enganche se han aplicado tres recubrimientos de acabado, Metaceram 28020, 28030 y 28060, también fabricados por Castolin, son polvos comerciales de tamaño de partículas entre $15 / 60 \mu \mathrm{m}$, con contenido de alúmina y titania $97 / 3,87 / 13$, y $60 / 40$ en peso, respectivamente.

Las condiciones de proyección para la capa de enganche y de los recubrimientos cerámicos están recogidas en la tabla I.

La proporción de porosidad y el tamaño máximo de poro se han estimado con análisis de imagen cuantitativo usando el analizador de imagen Omnimet Advantage, Image Análisis System de Buehler.

La porosidad del recubrimiento de alúmina/titania, medida en tres campos y expresando el valor medio, va reduciéndose al aumentar la proporción de titania, habiéndose encontrado una relación lineal, donde los valores de cada punto son el valor medio de tres campos, Figura 1.

$\mathrm{El}$ descenso de la temperatura de fusión del sistema $\mathrm{Al}_{2} \mathrm{O}_{3} /$ $\mathrm{TiO}_{2}$ según se incrementa el contenido de $\mathrm{TiO}_{2}$ en los polvos, facilita el desplazamiento de los gases y la reducción de la porosidad (4). La porosidad de la mezcla $60 / 40$ se considera baja para una proyección flame spray de $\mathrm{Al}_{2} \mathrm{O}_{3} / \mathrm{TiO}_{2}$ Los grandes poros son debidos a la formación de cavidades por insuficiente fusión, más bien que, por salpicaduras de las gotas proyectadas térmicamente (overlapping splats) (5). La estructura porosa resulta por, bajas temperaturas, bajo tiempo de residencia a la máxima temperatura, elevado tamaño de partículas del polvo, elevada velocidad de enfriamiento en función del grado de subenfriamiento y una relativamente alta velocidad de las partículas.
TABLA II. TAMAÑO DE PARTICULAS, TEMPERATURA DE FUSION Y DIAMETRO ESFERICO DE PORO.

\begin{tabular}{|c|c|c|c|c|}
\hline Muestra & $\begin{array}{c}\text { Tamaño de } \\
\text { partículas }\end{array}$ & $\begin{array}{c}\text { Temperatura } \\
\text { de fusión }\left({ }^{\circ} \mathrm{C}\right)\end{array}$ & $\begin{array}{c}\varnothing \text { esférico } \\
\text { de poro } \\
(\mu \mathrm{m})\left({ }^{*}\right)\end{array}$ & $\begin{array}{c}\mathrm{K}_{\mathrm{IC}} \\
\mathrm{MPa} \mathrm{m}^{1 / 2}\end{array}$ \\
\hline $97 / 3$ & $60-15$ & 2080 & 155 & 1,09 \\
\hline $87 / 13$ & $60-20$ & 2050 & 70 & 1,83 \\
\hline $60 / 40$ & $50-15$ & 1910 & 52 & 1,60 \\
\hline
\end{tabular}

$\left(^{*}\right)$ Es el diámetro esférico equivalente de todos los poros de la imagen.

Con el fin de poder identificar los cambios en las fases cristalinas desde el estado en polvo al estado proyectado, se han analizado por difracción de rayos $\mathrm{X}$ los tres materiales. Se ha utilizado un equipo Siemens D 5000 D, trabajando a temperatura ambiente con barridos de intensidad vs. ángulo de difracción en el rango $5-70^{\circ}$, velocidad de barrido $=3$ s/ skp usando radiación $\mathrm{K}_{\alpha}$ del $\mathrm{Cu}$ filtrado $(\lambda=1,5006 \AA$ ), un voltaje de $40 \mathrm{KV}$ y una corriente de filamento de $30 \mathrm{MA}$.

El material 97/3, Figura 2a, presenta los picos característicos del corindón, $\alpha$ - alúmina, tanto en el polvo como en la proyección, no apreciándose casi los débiles picos de la titania, pero en el recubrimiento proyectado se ha transformado parte de la $\alpha$-alúmina en $\gamma$-alúmina y $\delta$-alúmina, para las condiciones de proyección utilizadas. La alúmina estable romboédrica ( $\alpha$-alúmina o corindón) dependiendo de las condiciones de tratamiento térmico puede presentarse en forma de alúmina metaestable incluyendo la $\gamma$ (cúbica), $\delta$ (tetragonal u ortorrómbica), $\theta$ (monoclínica), K (ortorrómbica), $\eta$ (espinela cúbica), $\chi$ (espinela cúbica) (6-9).

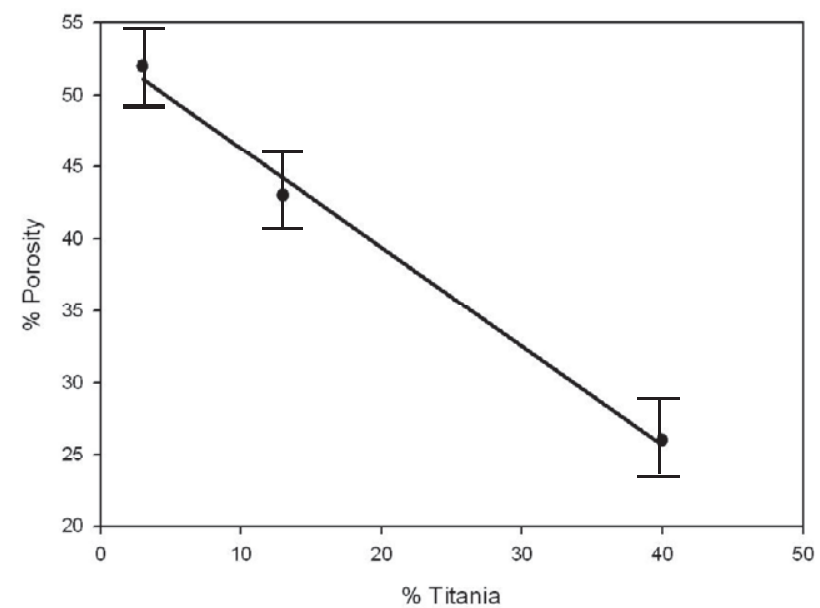

Fig. 1- Porosidad de los recubrimientos $\mathrm{Al}_{2} \mathrm{O}_{3} / \mathrm{TiO}_{2}$ en función del $\%$ $\mathrm{TiO}_{2}$ en peso. 
El material 87/13, Figura 2b, presenta los picos característicos de la $\alpha$-alúmina y de la titania, tanto en el polvo como en el depósito proyectado, no habiendo afectado las condiciones de proyección al tipo de fases presentes.

El material 60/40, Figura 2c, presenta picos característicos de la $\alpha$-alúmina y de la titania en el polvo, en cambio, en el recubrimientoproyectado sehatransformado mayoritariamente en la fase metaestable a temperatura ambiente $\mathrm{Al}_{2} \mathrm{TiO}_{5}$ con estructura tipo pseudobrookita (10).
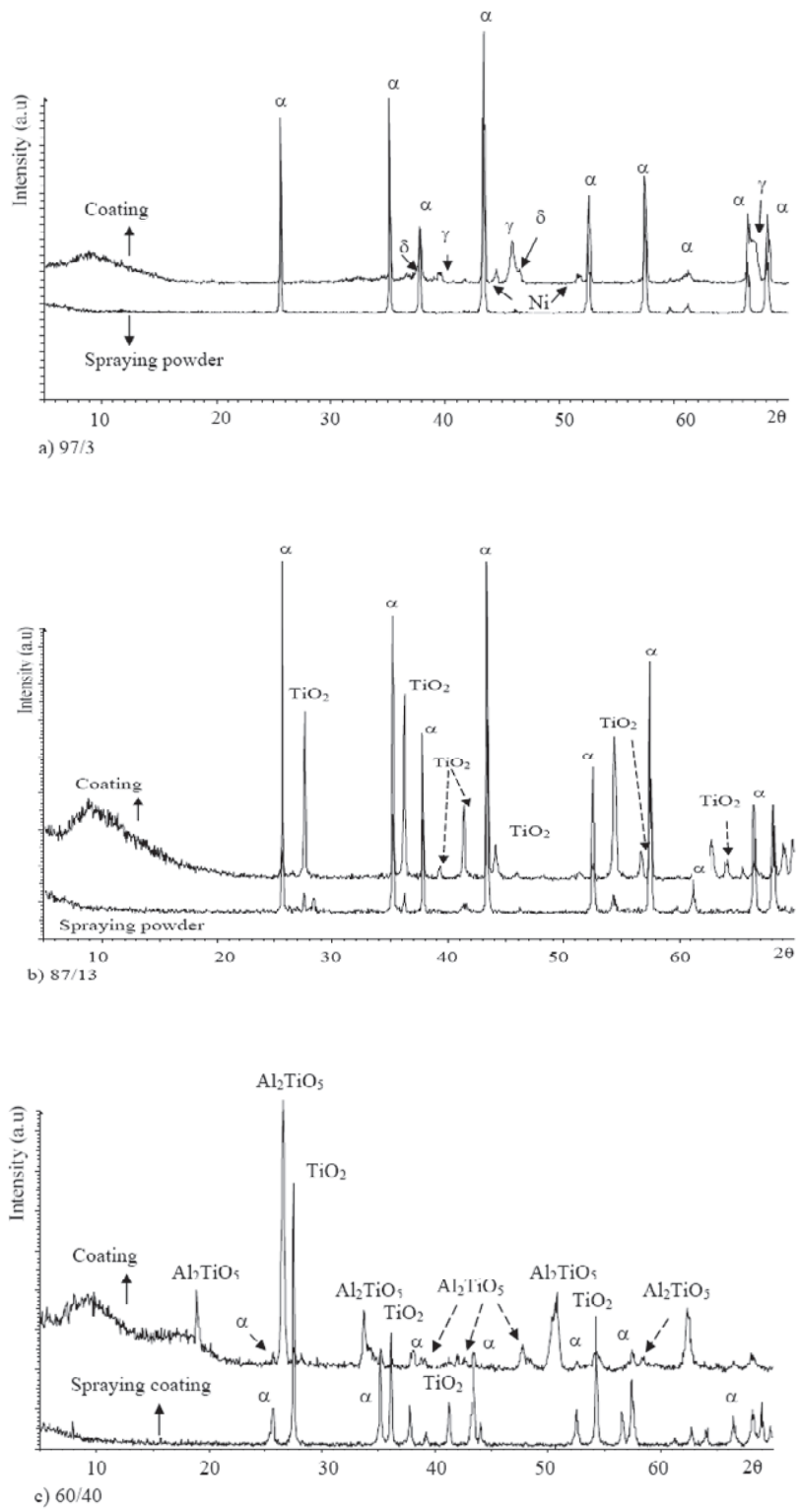

Fig. 2- Patrones de difracción de rayos X (XRD) de los polvo y de los recubrimientos proyectados alúmina/titania.

La alta sensibilidad de la $\alpha$-alúmina y de los sistemas alúmina/titania para producir distintas fases según el proceso de enfriamiento, la técnica y las condiciones de proyección térmica, la proporción de cada componente, e incluso la naturaleza de la capa de enganche, quedan puestas de manifiesto con la variación de estructuras obtenidas y consecuentemente sobre las propiedades (8).

\section{DUREZA}

La cara superficial y la sección transversal de todas las muestras fueron pulidas usando tres etapas secuenciales, de 30, 6 y $1 \mu \mathrm{m}$ de pasta de diamante antes de los ensayos. La dureza fue medida mediante indentaciones Vickers con carga de 200 gr durante 20 segundos sobre la superficie externa y en la sección transversal; cargas mayores difícilmente permiten distinguir los vértices de la huella dada la abundante porosidad. Se hicieron un mínimo de diez indentaciones por cada medida de dureza. Las grandes variaciones de dureza mostradas en la Figura 3, se deben al incremento del contenido en titania y las diferencias locales de dureza resultan de la variación de las temperaturas de las partículas y de las velocidades del proceso de proyección (11). La reducción de dureza en el recubrimeitno 60/40 es debida además a la formación de la fase $\mathrm{Al}_{2} \mathrm{TiO}_{5}$, cuya dureza es de $700 \mathrm{HV} / 100$. Se ha observado que cuando la carga del indentador se reduce, la microdureza aumenta debido a numerosos factores tales como recuperación elástica y efecto del borde de grano, ya que las dislocaciones alcanzan el borde de grano y endurece el material, siendo este efecto significativo cuando la huella de microdureza alcanza uno solo o pocos granos (12). Esta variación es mayor en materiales que no han recibido ningún tratamiento de estabilización (13-14).

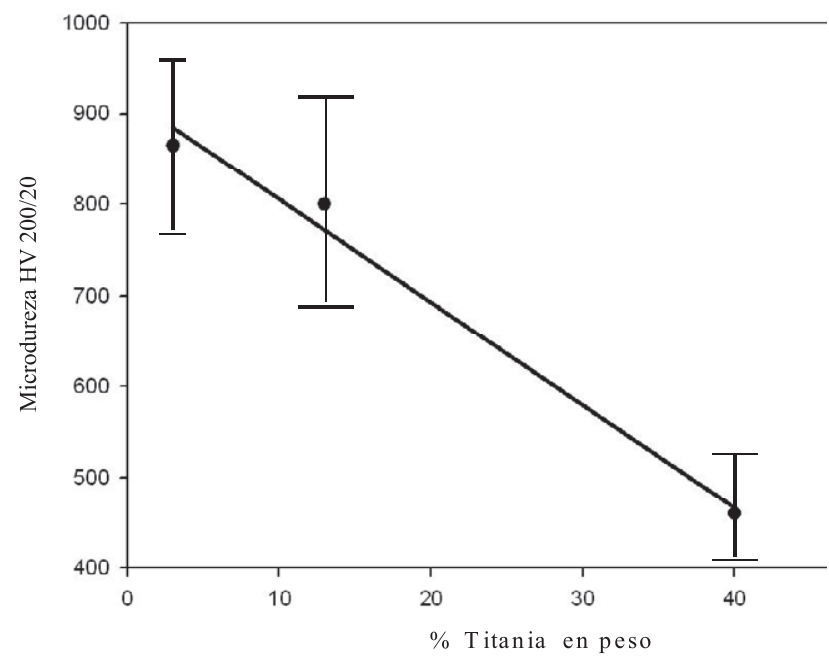

Fig. 3- Dureza de los recubrimientos cerámicos $\mathrm{Al}_{2} \mathrm{O}_{3} / \mathrm{TiO}_{2}$ en función del contenido en titania.

\section{TENACIDAD A FRACTURA}

Para medir la tenacidad a la fractura $\mathrm{K}_{\mathrm{IC}}$ en materiales cerámicos, hay varias técnicas experimentales. El uso de indentaciones de dureza Vickers, ha sido el método preferido debido a su simplicidad, y consiste en medir la longitud de las grietas que emanan de las indentaciones de la huella. Puesto que el trayecto tridimensional de las grietas no puede ser medido, el error es introducido en los cálculos y depende de las longitudes medidas de las grietas. De entre las diferentes fórmulas semiempíricas propuestas (15-20), el modelo utilizado ha sido el de Evans y Wilsham (20). 


$$
K_{I C}=\frac{0,079 P}{a^{3 / 2} \log \left(4,5 \frac{a}{c}\right)} \quad \mathrm{c} / \mathrm{a} \leq 4,5
$$

Donde $\mathrm{P}$ es la carga aplicada $(\mathrm{N}), \mathrm{c}$ es la longitud de la grieta desde el centro de la indentación y a es la mitad de la diagonal de la indentación.

Las medidas fueron realizadas tanto sobre las superficies como en sección donde el grosor del revestimiento varía entre $405-570 \mu \mathrm{m}$.

Los materiales en general, incrementan su tenacidad con el descenso de la dureza debido a que la deformación plástica es posible en el fondo de las grietas (21).

Como era de esperar la tenacidad a fractura del material con $13 \%$ de $\mathrm{TiO}_{2}$ es mayor que en el de $3 \%$, pero la muestra con $40 \% \mathrm{TiO}_{2}$ en vez de tener mayor tenacidad que el de $13 \%$ está en una posición intermedia. Se ha observado que mientras la fractura de los recubrimientos con 3\% y $13 \%$ $\mathrm{TiO}_{2}$ es transgranular a partir del vértice de la indentación, en el material con $40 \% \mathrm{TiO}_{2}$ la fractura es esencialmente intergranular, figura 4 a y b. El factor que puede afectar la tenacidad del material $40 \% \mathrm{TiO}_{2}$ es la composición química de los bordes de grano donde se ha detectado la presencia de un carburo mixto de Ti y Fe, que es duro y frágil y que debe influir negativamente en la tenacidad, Figura $5 \mathrm{a}$ y $\mathrm{b}$.

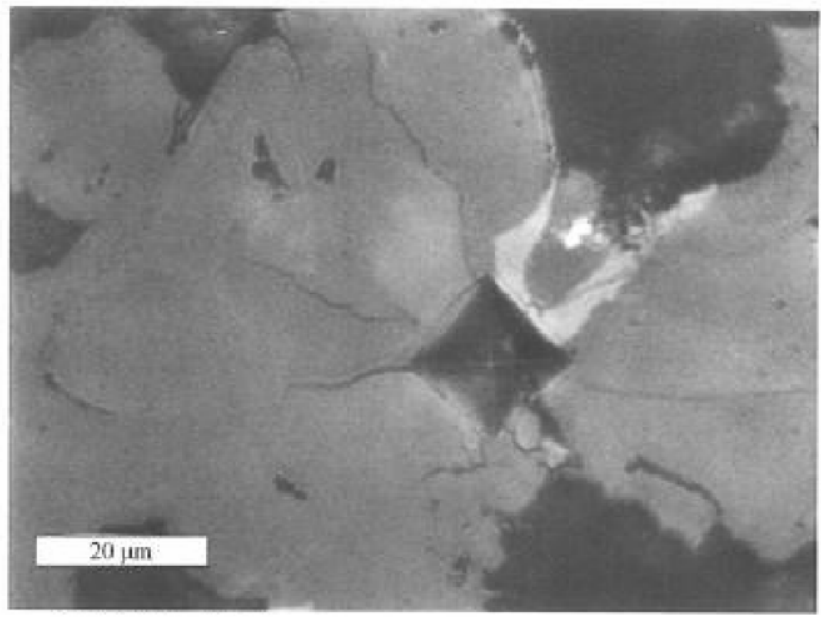

a) Grietas transgranulares en el recubrimiento $97 / 3$

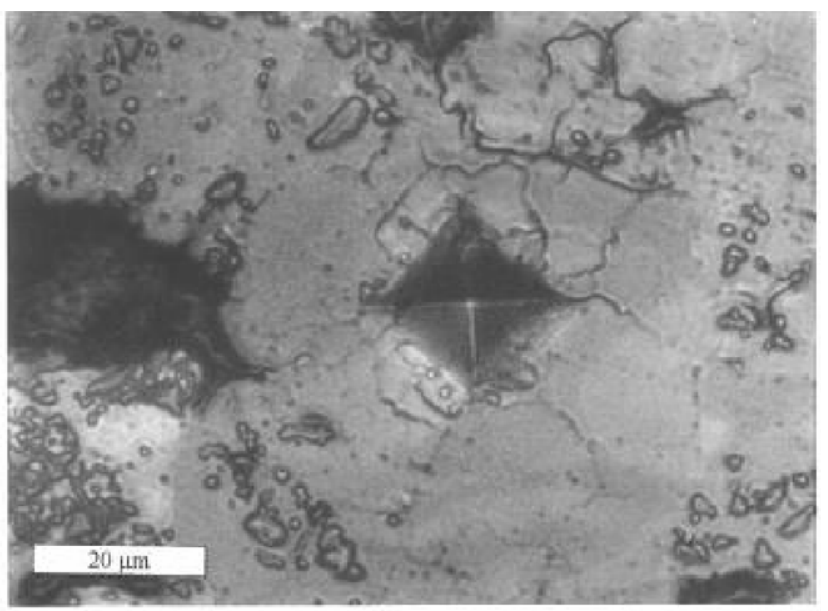

b) Grietas intergranulares en el recubrimiento $60 / 40$

Fig. 4- Indentaciones Vickers.

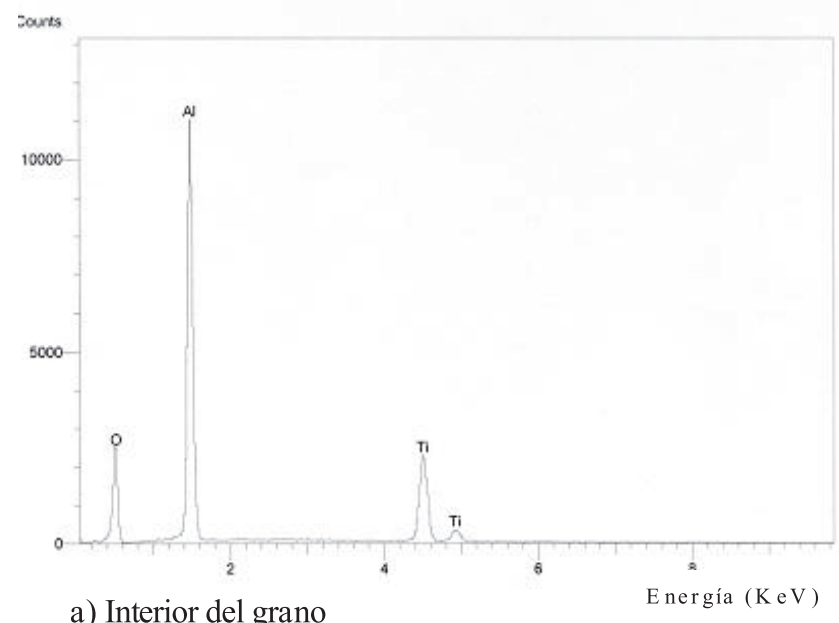

a) Interior del grano

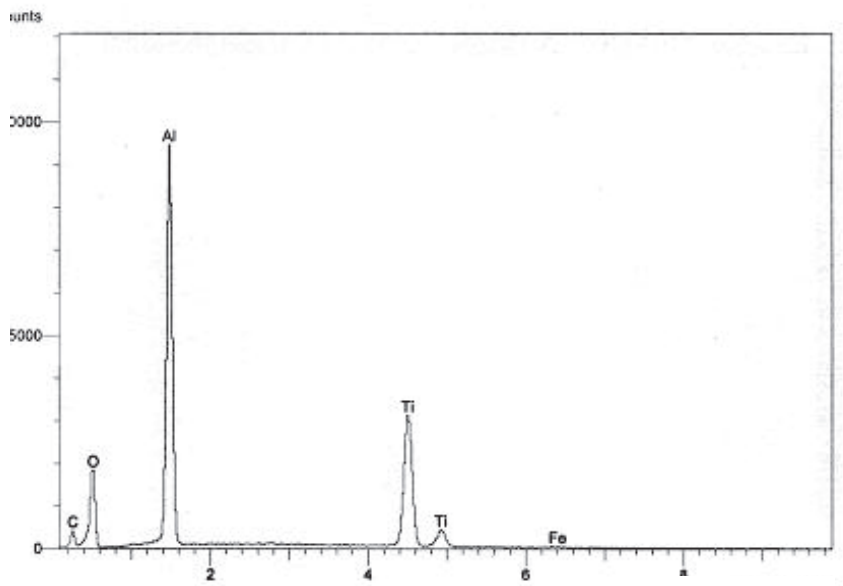

b) Borde del grano

Energía (Ke V)

Fig. 5- Análisis EDX del recubrimiento 60/40.

\section{DESGASTE ABRASIVO}

Los ensayos de desgaste se han desarrollado en un tribómetro multiaxial modelo pin on disc TE79/P de Plint \& Partners a temperatura y humedad ambiente. Las probetas a ensayar eran de acero inoxidable AISI 304 extraídas de una varilla de $8 \mathrm{~mm}$ de diámetro y fueron recubiertas en el extremo a ensayar por los recubrimientos cerámicos. Los anteriores pins se deslizaron bajo una carga de $5 \mathrm{~N}$ contra discos de abrasivos metalográficos de SiC P 240 de Buehler $(100 \mu \mathrm{m})$.

Las muestras hacían un recorrido de $9 \mathrm{~m}$ sobre el disco abrasivo con la finalidad de encontrar el abrasivo fresco durante el deslizamiento. El mantenimiento constante del coeficiente de rozamiento asegura que el abrasivo está fresco. Tres discos abrasivos, es decir $27 \mathrm{~m}$, fueron usados por cada probeta. La pérdida de material fue determinada pesando las muestras antes y después de cada cambio de disco abrasivo, y realizando una limpieza con ultrasonidos. Tres muestras fueron ensayadas por cada tipo de recubrimiento y sus resultados fueron reproducibles dentro de un $7 \%$. El desgaste abrasivo en $\mathrm{mg} / \mathrm{N}$.m se ha definido como la cantidad de 
material arrancada dividida entre la carga sobre la muestra y la distancia de deslizamiento. La inversa del desgaste abrasivo se define como la resistencia al desgaste abrasivo.

En este trabajo se ha determinado experimentalmente la resistencia al desgaste abrasivo y se ha comparado con la expresión propuesta por Liu Y. y otros (22), es decir:

$$
W R_{\text {abrasion }}=C \frac{H^{1 / 2} K^{2 / 3}}{(1+n P)}
$$

Donde $\mathrm{H}$ es la dureza, $\mathrm{K}$ es la tenacidad, $\mathrm{P}$ la porosidad, $\mathrm{n}$ un parámetro a fijar según los resultados experimentales. Estos valores están expresados por $\mathrm{m}$ de distancia de deslizamiento y por $\mathrm{N}$ de fuerza normal.

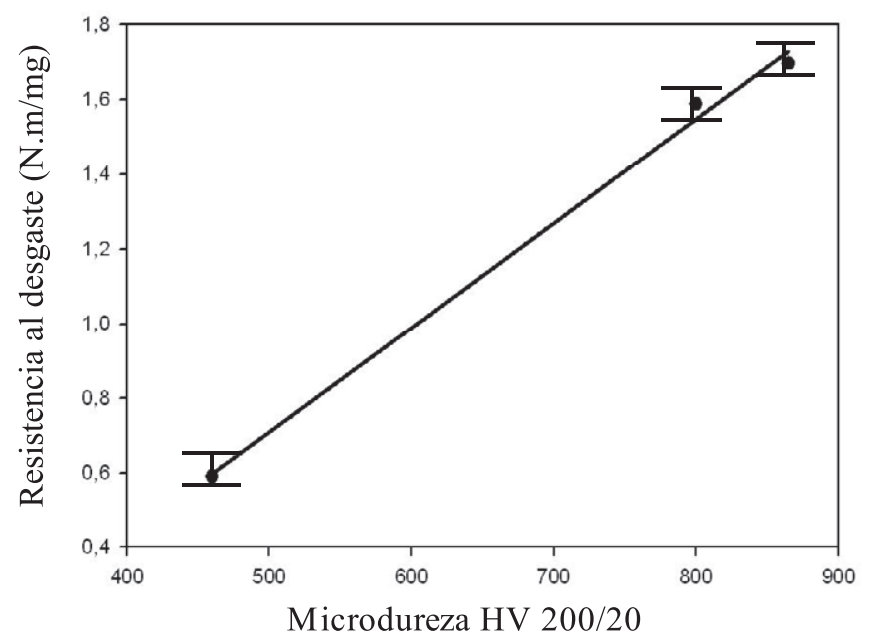

Fig. 6- Resistencia al desgaste abrasivo de los recubrimientos alúmina/titania en función de su dureza.

Se ha encontrado una pobre correlación entre los valores de resistencia al desgaste medidos experimentalmente y los calculados por la anterior expresión. De otra parte la Figura 7 , muestra que la relación entre la resistencia al desgaste y la dureza es lineal.

La información sobre el modo de desgaste es suministrada por micrografías electrónicas de barrido de la superficie y mediante el rayado realizado sobre los tres materiales con un equipo IPA-Scrach - Tester (Fraunhofer - Institut) con una carga de 5N, Figura 7. El desgaste en el material 97/3, muy duro y muy frágil, requiere fragmentar el material en pequeñas partículas para producir el arranque ya que la deformación plástica es prácticamente inexistente, lo que explica la mayor resistencia al desgaste. El desgaste en el material 87/13, algo menos duro y menos frágil ha supuesto fragmentar también el material pero con tamaños mayores de partículas y sin apreciarse signos de deformación plástica. Finalmente el material 60/40, con una dureza la mitad aproximadamente que el $97 / 3$, sufre desgaste con una fragmentación mucho menor y con signos de deformación plástica que se aprecian en el contorno de las partículas arrancadas, lo que justifica que su resistencia al desgaste sea mucho menor.

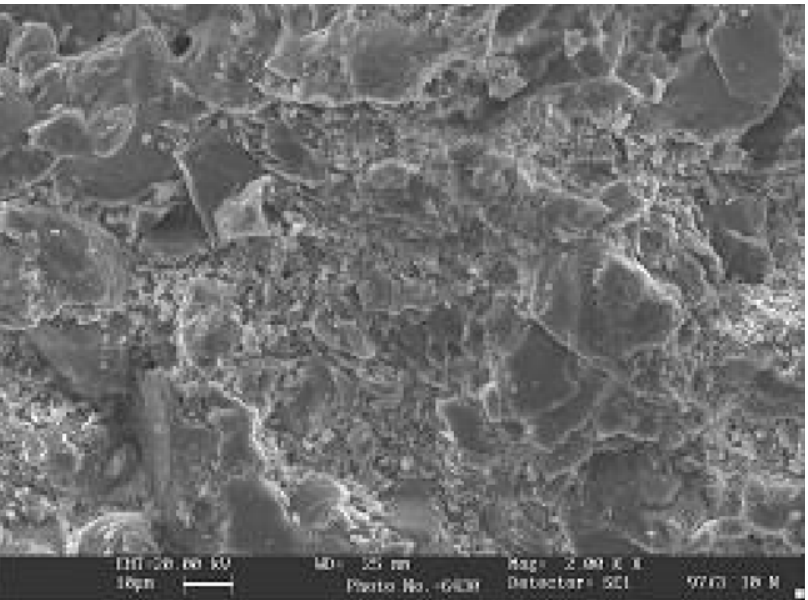

a) $97 / 3$

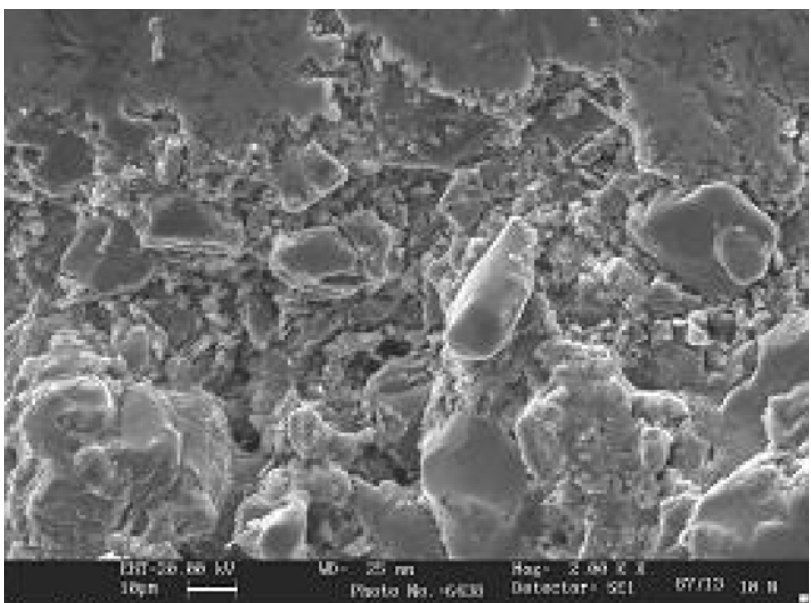

b) $87 / 13$

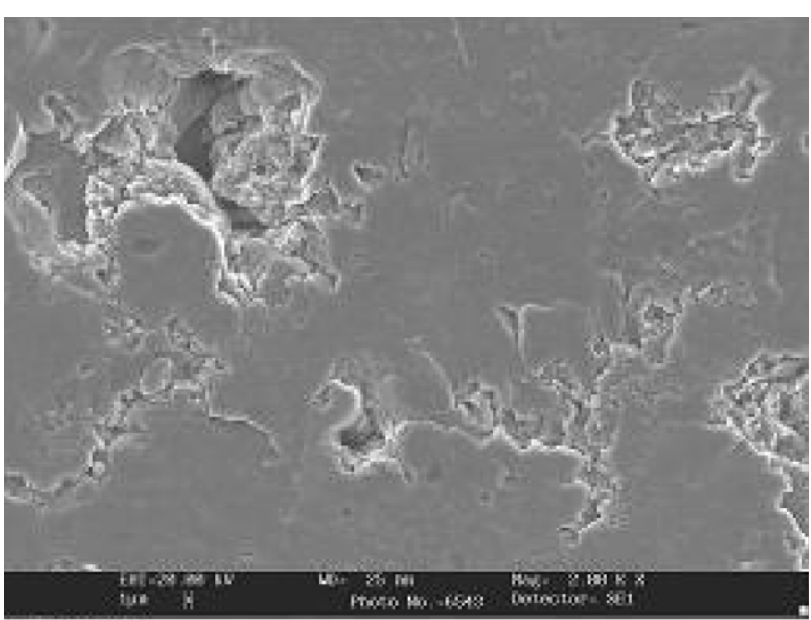

c) 6040

Fig. 7- Rayado de las superficies de los recubrimientos $\mathrm{Al}_{2} \mathrm{O}_{3} / \mathrm{TiO}_{2}$

\section{CONCLUSIONES}

- Se han estudiado tres recubrimientos alúmina/titania que han sido proyectados por oxyfuel en condiciones optimas y se ha encontrado una relación lineal inversa entre la porosidad y la proporción en peso de $\mathrm{TiO}_{2}$.

- Los sistemas $\mathrm{Al}_{2} \mathrm{O}_{3} / \mathrm{TiO}_{2}$ son muy sensibles a producir 
tras el proceso de proyección, distintas fases de alúmina así como el óxido mixto, según las variables del proceso.

- La dureza disminuye con el incremento de la proporción de $\mathrm{TiO}_{2}$ en peso.

- La resistencia al desgaste por abrasión de los recubrimientos es proporcional a su dureza.

\section{AGRADECIMIENTOS}

Los autores agradecen el soporte económico suministrado por la Fundación Caja Castellón - Bancaja, proyecto P1. B 2002 - 2B, y el apoyo durante los experimentos a D. José Ortega y $\mathrm{D}^{\mathrm{a}}$ Raquel Oliver del laboratorio de Ing. De Materiales y a D. Javier Gómez y D. Gabriel Perís de CSIC de la Universidad Jaume I de Castellón.

\section{BIBLIOGRAFÍA}

1. A. Kulkarni, S. Sampath, A. Goland, H. Hernan, Scr. Mater, 43 (2000) 471. 2. D.E Crawmer, Handbook of Termal Spray Technology, (2004) 55.

3. N.Wagner, K.Gnädig, H.Kreye, H. Kronewetter, Surf. Technol. 22 (1984) 61. 4. D. Golberg, Rev. Int. Hautes Tem. Refract. 5 (3) (1968) 181.

5. Y.R. Liu, Y. F. Qiao, J. He, E. J. Lavernia, T.E. Fisher, Metall. Mater. Trans. A (2002) 33.

6. I. Levin, L.A: Bendersky, D. G. Brandon, M. Rühle, Acta Mater. 45 (9) (1997), 3659.

7. P.S. Santos, H. S. Santos, S.P Toledo, Mater. Res. 3 (4) (2000) 104.

8. M. Uma, Ceram. Intern. 30 (2004) 555

9. G. Paglia, Thesis, Curtin University of Techn. (2004).

10. B.Morasin, R.W.Lynch, Acta Cristallogr. B 28 (1972) 1040.

11. M.Birkholz, U.Albers, T.Jung, Surf. Coat. Technol. 179 (2004) 279.

12. S.J. Bull, D.S. Richerby, Avd. Surf. Coat., (1991) 315.

13. G.D.Quinn, Indentation Hardness Testing of Ceramics, Mechanical Testing and Evaluation, ASM Handbook, vol 8., p 244.

14. J.B. Quinn, G. D. Quinn, J. Mater. Sci., 32 (1997) 4331.

15. G.R. Austis, J. Am. Ceram.. 64 (9) (1994), 533.

16. G.K. Berhish, C.W. Florey, F. J. Worzala, W.T. Lenhing, J. Therm. Spray Technol. 2 (1) (1993) 35.

17. H.M. Hawthorne, L. C. Erickson, D. Ross, H. Tai, T. Troczynski, Wear 203/204 (1997) 709.

18. K.M. Liang, J. Mater. Sci. 25 (1990), 207.

19. P Ostojic, R Mac Pherson, Mater. Forum 10 (4) (1987),247.

20. A. F. Evans, T.R. Wilshaw, Acta Met. 24 (1976), 939.

21. J.Meza, J.Chaves Dyna vol 70, 139 (2003) 53.

22. Y. Liu, T. Fischer, A. Dent. Surf. Coat. Tech. 176 (2003), 68.

Recibido: 06.11 .06

Aceptado: 27.11 .07 